\title{
On the creep behavior of carbon/epoxy non-crimp fabric composites
}

\author{
Natália Pagnoncelli Lorandi ${ }^{a}$, Maria Odila Hilário Cioffi ${ }^{b}$ Carlos Shigue ${ }^{c}$, Heitor Luiz Ornaghi Jr. ${ }^{a}$
}

\author{
${ }^{a}$ Programa de Pós-Graduação em Engenharia e Ciência dos Materiais (PGMAT), Universidade de \\ Caxias do Sul (UCS), 95070-490, Caxias do Sul, RS, Brasil \\ ${ }^{b}$ Grupo de Fadiga e Materiais Aeronáuticos, Universidade Estadual Paulista (UNESP), 12516-410, \\ Guaratinguetá, SP, Brasil \\ ${ }^{c}$ Escola de Engenharia de Lorena (EEL), Universidade de São Paulo (USP), 12602-810,
}

Lorena, SP, Brasil

Received: August 27, 2017; Revised: January 08, 2017; Accepted: January 18, 2018

\begin{abstract}
The use of composites in structural applications requires the study of specific properties to ensure that these materials are safe and an excellent option to replace some metals, for example. In the present study, vacuum assisted resin transfer molding (VARTM) was used prior to obtain carbon/epoxy noncrimp fabric composites. Dynamic mechanical analysis and creep - by using Findley and Burger's methods - tests were performed aiming to evaluate the viscoelastic properties of the composites, in three different temperatures $\left(30,60\right.$ and $\left.90^{\circ} \mathrm{C}\right)$ and static stresses $(2,5$ and $10 \mathrm{MPa})$. The viscoelastic properties were also discussed using Weibull parameters obtained from creep curves. In general, the deformation was temperature and stress dependent, which was corroborated by the viscoelastic parameters obtained from both models.
\end{abstract}

Keywords: Non-crimp fabric (NCF), Creep behavior, Dynamic mechanical analysis, viscoelasticity.

\section{Introduction}

Carbon/epoxy composites are lightweight materials with high specific properties, such as strength and stiffness. These materials are widely used in structural applications, especially in replacement of metals and metallic alloys in airplane parts such as wings and fuselage panels ${ }^{1,2}$. To enhance the performance of these materials, fiber non-crimp fabrics (NCF), have been gaining attention due some advantages as: easy to handle and store, improved out-of-plane properties, and maximization of resin migration through layers, in comparison with woven fabrics ${ }^{3-5}$. Besides material selection, molding technique also plays an important role in the final characteristic of the composite. Vacuum assisted resin transfer molding (VARTM) process shows advantages over some liquid molding processes as resin film infusion (RFI), because the molding system is made with flexible bags and vacuum is applied to enhance resin flow and decrease void content into final parts produced. The result is composites with better mechanical properties in comparison with those produced with rigid molding systems ${ }^{1}$. Since the main characteristics of VARTM and resin film infusion (RFI) are identical, one of the main differences is that in VARTM the thickness is controlled by the mold tooling cavity, while in the infusion process a part with a thickness is controlled by the fibers' compaction under vacuum. Another important difference is that in RFI process, resin must be storage under low temperature (to maintain solid format) prior to use ${ }^{6}$.
It is well known that the composite properties after processing depends on the fiber/matrix interface, which is the main component of the stress transfer. There are many direct and indirect manners to calculate and estimate the interface of a composite material. For example, the dynamic mechanical and creep behaviors are two of indirect ones, which are very interesting since they allow to predict the modulus and strength over time and temperature ${ }^{6,7}$. Since majority of composite properties are highly dependent of interface quality, in using small stresses by a dynamic mechanical analyzer, due equipment sensibility, any creep, which will occur, on sample will be due resin flow, mainly on interface. So, interface can be estimated, in this case, as a quantitatively tool in comparing a pack of samples under any allowed stress level by the apparatus. In addition, since the final product will experience a range of temperatures/ stresses, it becomes important to predict how the final product will behave under such conditions ${ }^{8}$.

In literature, there are few reported studies about creep $v s$ structure-property relationship of composite materials, mainly in applying low stress profile. In one of them, creep behavior from Findley and Burger models was studied by Yang ${ }^{9}$ aiming to understand the structure-property based on both parameters. The authors studied polyamide 66 (PA 66) with titanium dioxide $\left(\mathrm{TiO}_{2}\right)$ nanoparticles with and without superficial treatment and PA66/clay nanocomposites. Burger parameters were studied and related to the rigidity 
of the composite, amorphous and crystalline regions of the thermoplastic matrix, elastic and permanent deformation of the composite and interaction between nanoparticles and matrix. Maxwell and Kelvin modulus $\left(E_{m}, E_{k}\right)$ as well Kelvin viscosity $\left(\eta_{K}\right)$ showed to be dependent of the stress and temperature, indicating that the materials behavior with higher rigidity due nanoparticles incorporation. Maxwell viscosity $\left(\eta_{M}\right)$ was more influenced by nanoparticles addition, with lower creep deformation. In other study Militky and Jabbar ${ }^{10}$ studied jute/epoxy composites aiming to verify the influence of the fiber treatment methods. The test temperatures were selected below the glass transition temperature from the loss modulus by a dynamic mechanical analyzer. The authors used Burger model to evaluate the relation between morphology and creep behavior. All parameters decrease with temperature, independently of the superficial treatment applied. They also related the results with modifications in the rigidity molecular mobility of the matrix chains and to the fiber/matrix adhesion. Discrepancies presented on dynamic-mechanical results were associated to the matrix/ fiber interface, since resin, fiber volume and orientation were similar. In similar study, Almeida Jr. et al. ${ }^{11}$ analyzed the creep and interfacial behavior of carbon fiber reinforced epoxy filament wound laminates at five different winding sequences. The authors used two different analytical models for it (Findley and Burger's models). The author verified that viscoelastic behavior is dependent of the fiber orientation as well the creep performance. Both Burger and Findley's analytical models were suitable to predict creep behavior, showing good fitting of the experimental data. But, at higher temperatures, a larger difference in fitting is observed and as the ply angle increases, Findley's seems to adjust to the experimental data better than Burger's at higher temperatures. In general, Findley's power law is simpler and can be satisfactory to express long-term creep properties, whereas Burger's provides a more constitutive model, and allows a more detailed structure-to-property relationship analysis, with a poorer fitting at higher temperatures, in this case. In other study, Goertzen and Kessler ${ }^{12}$ evaluated creep behavior of carbon/epoxy composites under tensile and flexural loadings and the results suggested that the composite would not fail below $65 \%$ of the tensile strength for $1,600 \mathrm{~h}$. However majority of the studies performing creep use high stress levels which is necessary an expensive equipment and higher analyses cost. In using a more sensitive equipment, as a DMA, lower stress levels are allowed and it is possible to obtain more accurate information in respect to structure of the composite in study as structural changes are occurring.

The present study has as novelty a detailed structureproperty relationship study on viscoelastic properties of carbon NCF/epoxy composites molded by VARTM. The viscoelastic properties of the composite were evaluated by dynamic mechanical analysis and by Findley, Burgers and
Weibull model parameters using creep testing based on literature ${ }^{11-14}$. Different conditions of temperature and static stress were selected aiming to evaluate the behavior along time on creep tests.

\section{Materials and Methods}

\subsection{Materials and composites processing}

A carbon non-crimp fabric (NCF) (Saertex, with Hexcel carbon fibers IM7 12K, GP) was used as reinforcement in composite materials. Fabrics were arranged in 16 layers in a quadraxial architecture of $\left[(0,90, \pm 45)_{\mathrm{S}}\right]_{2}$. The fiber volume content in each composite was $60 \pm 1 \%$ and the void content was $3.4 \pm 0.3 \%$, both determined by acid digestion (ASTM D3171-15). Density of composites was $1.53 \pm 0.02$ g. $\mathrm{cm}^{-3}$, determined according to the Archimedes principle (ASTM D792).

The epoxy resin chosen was PRISM ${ }^{\mathrm{TM}}$ EP2400 (Cytec Engineered Materials). Before processing, the liquid resin was vacuum degassed to remove air bubbles, and along the process, vacuum of $5.5 \times 10^{-2} \mathrm{mPa}$ was applied into the mold, to prevent voids and to assist resin transfer. The temperature of the mold and injection was maintained at $100^{\circ} \mathrm{C}$ and cure was performed at $180^{\circ} \mathrm{C}$ for $2 \mathrm{~h}$, also in the mold. The final composite had about $3 \mathrm{~mm}$ thickness.

\subsection{Characterization of the composites}

Dynamic mechanical properties of composites were measured by three-point bending mode using a dynamic mechanical analyzer DMA Q-800 of TA instruments. The testing conditions were controlled in the temperature range of $25-250^{\circ} \mathrm{C}$, fixed frequency of $1 \mathrm{~Hz}$, preload of $0.1 \mathrm{~N}$, amplitude of $20 \mu \mathrm{m}$, force track of $125 \%$. Two dynamic runs were performed to assure that both composites were compared in the same conditions ${ }^{9}$ : the first run at a heating rate of $10^{\circ} \mathrm{C} \cdot \mathrm{min}^{-1}$, to eliminate interface effects; and the second run at $3^{\circ} \mathrm{C} \cdot \mathrm{min}^{-1}$, which is more sensitive to relaxation processes.

Creep tests were also conducted on the same equipment as DMA within the temperature of $30-90^{\circ} \mathrm{C}$ in step of $30^{\circ} \mathrm{C}$. The temperature range was selected below the glass transition temperature (obtained from the loss modulus curves) of composites ${ }^{10}$. Static stresses of 2, 5 and $10 \mathrm{MPa}$ were applied at the center point of long side of the sample through the sample thickness for $30 \mathrm{~min}^{10}$. The static stress was selected after performing a strain sweep test, where the linear viscoelastic region was defined ensuring that creep tests were conducted in the linear region. Findley, Burger's and Weibull models were fitted with Nonlinear Curve Fit analysis at OriginPro 8.1 software.

Samples of $3 \mathrm{~mm}$ thickness, $10 \mathrm{~mm}$ width and $50 \mathrm{~mm}$ length were used for DMA and creep. 
Findley and Burger's models were used aiming to make a structure-property relationship of the composites. Findley's model consists of an empirical equation (Eq. 1) that describes with good precision the viscoelastic behavior of polymers and polymeric composites in a large time scale:

$$
\varepsilon(t)=\varepsilon_{0}+A t^{n}
$$

where $\varepsilon(t)$ is the creep strain, $t$ is the time, $\varepsilon_{0}$ is the time-independent strain; $A$ is the amplitude of transient creep strain, a time-dependent term; and $n$ is a constant, with values usually less than $1^{9,15}$.

Burger's model is based on a combination in series of a Maxwell with a Kelvin-Voigt element [Eq. 2 and 3]. The total creep strain is divided into three separate parts: the instantaneous elastic deformation (Maxwell spring, $\varepsilon_{M}$ ), viscoelastic deformation (Kelvin unit, $\varepsilon_{K}$ ) and viscous deformation (Maxwell dash-pot, $\varepsilon_{\infty}$ ). Thus, total strain as a function of time can be represented by the following equations:

$$
\begin{gathered}
\varepsilon(t)=\varepsilon_{M}+\varepsilon_{K}+\varepsilon_{\infty} \\
\varepsilon(t)=\frac{\sigma_{0}}{E_{M}}+\frac{\sigma_{0}}{E_{K}}\left(1-e^{-E_{K} t / \eta_{K}}\right)+\frac{\sigma_{0}}{\eta_{M}} t
\end{gathered}
$$

where $\varepsilon(t)$ is the creep strain, $\sigma_{0}$ is the applied stress, $t$ is the time, $E_{M}$ and $E_{K}$ are the elastic moduli of Maxwell and Kelvin springs, respectively, $\eta_{M}$ and $\eta_{K}$ are respectively, the viscosities of Maxwell and Kelvin dashpots. $\eta_{K} / E_{K}$ is also denoted as $\tau$, the retardation time required to generate $63.2 \%$ deformation in the Kelvin unit. The first term of Eq. 2, $\varepsilon_{M}$, is a constant value and does not change with time. $\varepsilon_{K}$ represents the earliest stage of creep and attains a saturation value in short time and $\varepsilon_{\infty}$ represents the trend in the creep strain at sufficiently long time, and appears similar to the deformation of a viscous liquid obeying Newton's law of viscosity9.

As an earlier study proposed by Ornaghi jr et al. ${ }^{16}$ the width of the $\tan \delta$ curve can be someway related to the $\beta$ stretched exponential, which give us an idea of the distribution of relaxation times. Lower $\beta$ values will imply in a wider distribution; higher $\beta$ values will represent a narrow one. Base on this, an alternative method for calculate $\beta$ is to apply the latch-based Weibull model for polymeric creep [Eq. 4$]^{8}$ that can link both analyses:

$$
\varepsilon(t)=\varepsilon_{i}+\varepsilon_{c}\left[1-e^{\left(-t / \eta_{c}\right)^{\beta_{c}}}\right]
$$

where: $\varepsilon(t)$ is the total strain over time, $\varepsilon_{i}$ is the instantaneous strain from initial application of the load, $\varepsilon_{c}$ is the creep strain, $\eta_{c}$ is the characteristic lifetime and $\beta_{c}$ is the shape parameter.

\section{Results and Discussion}

\subsection{Dynamic mechanical properties}

Figure 1 shows the dynamic mechanical curves as a function of the temperature for VARTM carbon NCF/epoxy composite. Curves are referred to the second run ${ }^{17}$.

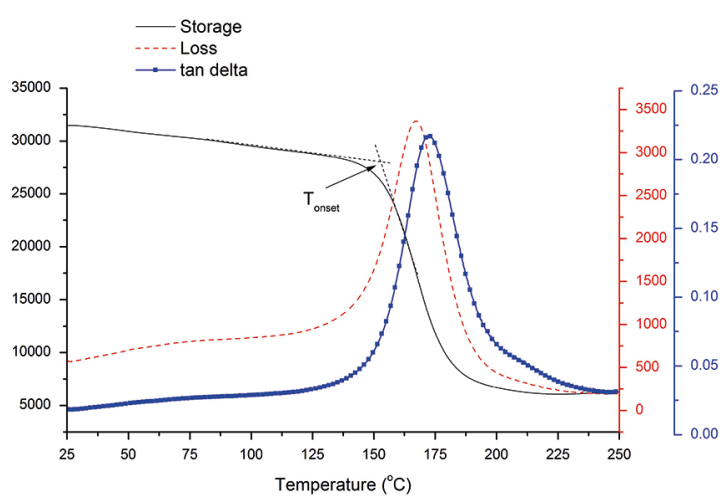

Figure 1. Storage modulus, loss modulus and $\tan \delta$ curves for carbon NCF/epoxy composites produced by VARTM.

For aeronautic applications, maybe the most important curve is the storage modulus, since it represents purely the mechanical properties which the part can take after a load (as tensile or flexure tests, for example) $)^{18}$. The storage modulus at $30^{\circ} \mathrm{C}$ had shown the following result: $28.8 \mathrm{GPa}$ for VARTM process composite with $\mathrm{T}_{\text {onset }}$ of $144^{\circ} \mathrm{C}$.

The maximum loss modulus was $3.14 \mathrm{GPa}$ at $167^{\circ} \mathrm{C}$. The $\tan \delta$ conveys no physical magnitude but it is very important to determine some macroscopic physical properties as the damping of free vibrations, the attenuation of propagated waves and the frequency width of a resonance response ${ }^{19}$. Besides the maximum energy dissipation point, the height and the width of the curve contains important information about the composite material. In this study, $\tan \delta_{\max }$ value was 0.285 at $172^{\circ} \mathrm{C}$. The narrow curve presented by the composite can be related to the distribution of the relaxation times related to the cooperativity of the system. The better the interaction fiber/matrix, the higher inhibition of the relaxation process, because the matrix will take more time to relaxation occur, leading also to a broader temperature range $\mathrm{e}^{20,21}$.

\subsection{Creep measurement}

Figure 2 shows the creep curves for the composites studied at different temperatures and stresses with Findley and Burger's fits. The curves showed a typical behavior presented by Findley's ${ }^{15}$. It can be noted that the deformation was higher for VARTM composites tested in higher temperature and static stresses which corroborated with literature ${ }^{9,10}$. The data for Findley's parameters are presented in Table 1. 

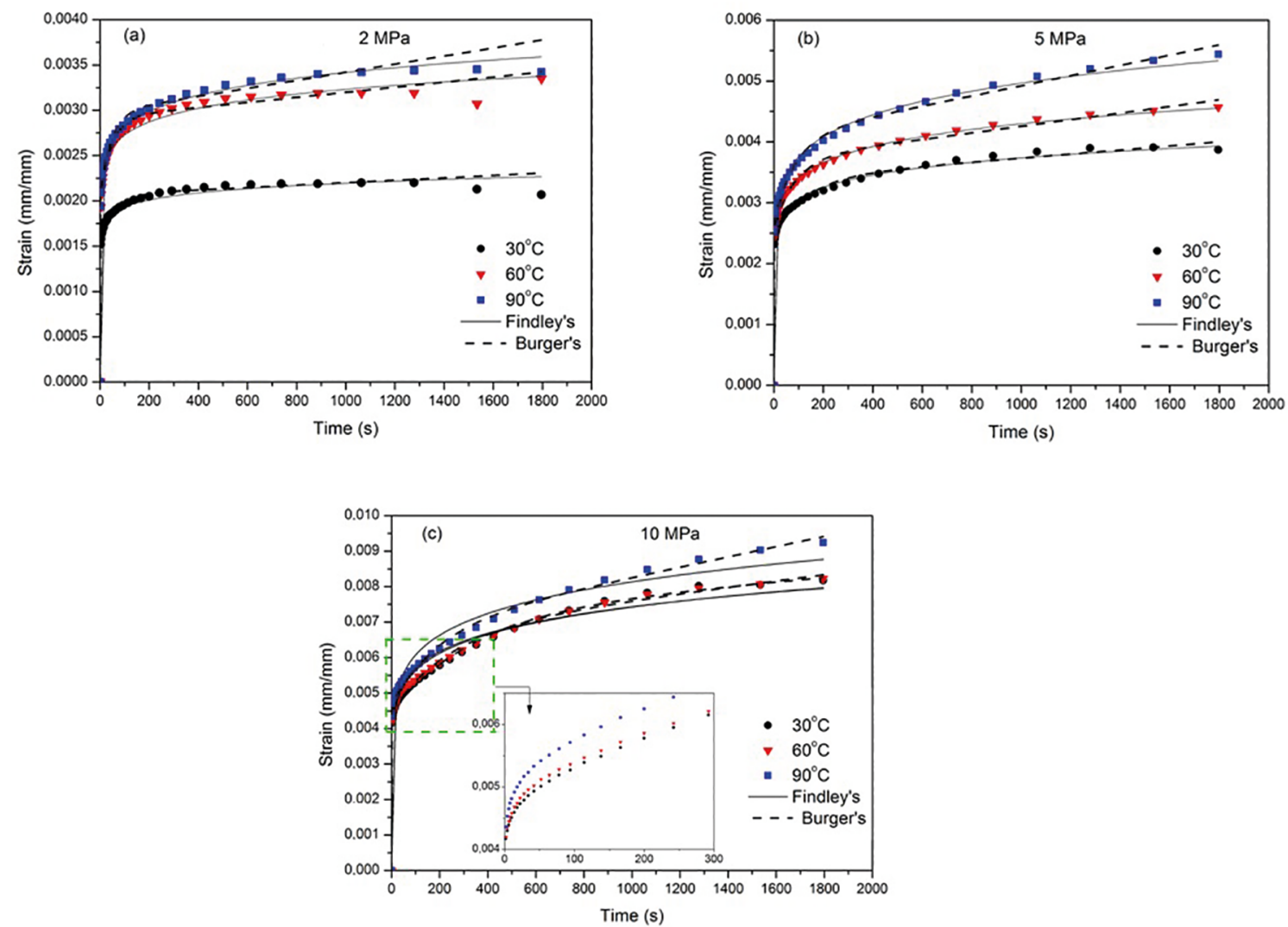

Figure 2. Creep curves and applied models of carbon NCF/epoxy composites at(a) 2 (b) 5 and (c) $10 \mathrm{MPa}$.

Table 1. Findley's parameters for carbon/epoxy NCF composite, at different stresses.

\begin{tabular}{cccccc}
\hline \multirow{2}{*}{ Stress (MPa) } & Temp. $\left({ }^{\circ} \mathrm{C}\right)$ & \multicolumn{3}{c}{ Findley's parameters } \\
\cline { 3 - 6 } & $30^{\circ} \mathrm{C}$ & $1.51 \times 10^{-3}$ & 0.0547 & $\varepsilon_{0}\left(m m \cdot m m^{-1}\right)$ & $\mathrm{R}^{2}$ \\
\hline \multirow{2}{*}{2} & $60^{\circ} \mathrm{C}$ & $1.96 \times 10^{-3}$ & 0.0733 & $-0.57 \times 10^{-5}$ & 0.983 \\
& $90^{\circ} \mathrm{C}$ & $1.93 \times 10^{-3}$ & 0.0833 & $-1.63 \times 10^{-5}$ & 0.987 \\
& $30^{\circ} \mathrm{C}$ & $2.05 \times 10^{-3}$ & 0.0863 & $1.68 \times 10^{-5}$ & 0.995 \\
5 & $60^{\circ} \mathrm{C}$ & $2.15 \times 10^{-5}$ & 0.0996 & $1.77 \times 10^{-5}$ & 0.995 \\
& $90^{\circ} \mathrm{C}$ & $2.10 \times 10^{-3}$ & 0.1230 & $5.49 \times 10^{-5}$ & 0.998 \\
& $30^{\circ} \mathrm{C}$ & $3.08 \times 10^{-3}$ & 0.1235 & $20.3 \times 10^{-5}$ & 0.996 \\
& $60^{\circ} \mathrm{C}$ & $3.20 \times 10^{-3}$ & 0.1183 & $17.3 \times 10^{-5}$ & 0.970 \\
& $90^{\circ} \mathrm{C}$ & $3.17 \times 10^{-3}$ & 0.1317 & $25.9 \times 10^{-5}$ & 0.975 \\
\end{tabular}

In general, Findley's parameters tend to increase by increasing temperature from 30 to $90^{\circ} \mathrm{C}$, because these parameters are related with creep strain, which also increases with temperature. However, no clearly tendency was observed on these parameters. The model's curve did not fit as well as Burger's one for the composite and for all temperatures. So, since Findley's model (three parameters) is not so sensitive, Burger's (four parameters) was used aiming to make a structure property relationship. Also, negative values were obtained in using $2 \mathrm{MPa}$ for the time-independent strain parameter.
Since this parameter is related to the initial strain (negative values could be erroneous interpreted in terms of negative deformation), in our case, can be due the load stress level is too low to promote any significant deformation, even leading out to negative values (due mainly by the Findley's model fitting procedure).

Table 2 presents the four parameters of Burger's models for VARTM composites. It is also presented the retardation time, $\tau$. All parameters will be discussed separately. 
Table 2. Burger's parameters for carbon/epoxy NCF composite, at different stresses.

\begin{tabular}{ccccccccc}
\hline \multirow{2}{*}{ Stress (MPa) } & Temp. $\left({ }^{\circ} \mathrm{C}\right)$ & \multicolumn{7}{c}{ Burger's parameters } \\
\cline { 3 - 8 } & & $E_{M}(\mathrm{MPa})$ & $E_{K}(\mathrm{MPa})$ & $\eta_{K}(\mathrm{MPa} . \mathrm{s})$ & $\eta_{M}(\mathrm{MPa} . \mathrm{s})$ & $\mathrm{R}^{2}$ & $\tau(\mathrm{s})$ \\
\hline \multirow{2}{*}{2} & $30^{\circ} \mathrm{C}$ & 1263 & 4129 & $2.46 \times 10^{5}$ & $15.0 \times 10^{6}$ & 0.982 & 59.6 \\
& $60^{\circ} \mathrm{C}$ & 980 & 2282 & $1.06 \times 10^{5}$ & $7.12 \times 10^{6}$ & 0.980 & 46.3 \\
& $90^{\circ} \mathrm{C}$ & 970 & 2192 & $0.99 \times 10^{5}$ & $4.49 \times 10^{6}$ & 0.981 & 45.3 \\
5 & $30^{\circ} \mathrm{C}$ & 2026 & 5382 & $7.00 \times 10^{5}$ & $14.9 \times 10^{6}$ & 0.983 & 130.1 \\
& $60^{\circ} \mathrm{C}$ & 1932 & 4448 & $3.83 \times 10^{5}$ & $9.21 \times 10^{6}$ & 0.988 & 86.2 \\
& $90^{\circ} \mathrm{C}$ & 1843 & 3651 & $3.27 \times 10^{5}$ & $5.96 \times 10^{6}$ & 0.990 & 89.6 \\
& $30^{\circ} \mathrm{C}$ & 2242 & 3646 & $12.3 \times 10^{5}$ & $16.9 \times 10^{6}$ & 0.993 & 336.5 \\
& $60^{\circ} \mathrm{C}$ & 2217 & 4489 & $11.2 \times 10^{5}$ & $11.3 \times 10^{6}$ & 0.992 & 250.1 \\
& $90^{\circ} \mathrm{C}$ & 2125 & 4726 & $9.30 \times 10^{5}$ & $6.94 \times 10^{6}$ & 0.992 & 196.8 \\
\hline
\end{tabular}

\subsubsection{Time-independent elasticity, $E_{M}$}

This parameter represents the Maxwell spring in the instantaneous creep strain, which could be immediately recovered on the removal of stress. $E_{M}$ is dependent of temperature because the bulk material tends to become softer with temperature, which decreases the instantaneous modulus. Since this parameter is sensitive to the ability of load bearing and to the elastic stiffness of the materials, it can be associated with storage modulus in the glassy region, where higher temperature decreases the modulus when compared same static stress applied ${ }^{9}$.

The results suggest that it is difficult to viscous flow of bulk materials due a more elastic deformation in the short-time response. Therefore, more cooperativity is necessary for higher stresses, the enhanced mobility of amorphous chain is harder to achieve, which increase the time-independent elasticity values by static stress applied. The higher the stress applied, the higher will be the resulting time-independent elasticity, since higher stress applied reflects on higher modulus ${ }^{22}$. For semicrystalline polymers, this parameter can be related to the elasticity of the crystallized zones when are subjected to immediate stress due to their higher stiffness ${ }^{22}$. In our case, it can be related to the elasticity stiffness of the amorphous region, which increases according to the Hooke's law.

\subsubsection{Retardancy elasticity $E_{K}$ and viscosity $\eta_{K}$}

These parameters can be associated with the stiffness and viscous or orientated flow of amorphous polymer chains in short term, respectively ${ }^{9}$. The retardancy elasticity $E_{K}$ and viscosity $\eta_{K}$ showed similar dependency on temperature and stress. When the temperature increases and tends to achieve the glass transition, amorphous polymer became much active and was promptly oriented in a short-term period once the stress loaded. The viscous slippage of the molecules becomes easier to achieve upon temperature. So, $E_{K}$ and $\eta_{K}$ decreased by increasing temperature due to greater energy absorption by the active polymer chains; consequently the stiffness decreases. Also, the long-chained molecules are not able to withstand deformation at higher temperatures as they become increasingly active due to the greater energy absorption ${ }^{9,22}$. In general, $E_{K}$ and $\eta_{K}$ were considerably increased with increasing load, showing high stress dependency. Again, due higher stress applied, the response of the molecular chains relaxation will be higher, leading out to higher values. Polymer chains thereafter lied in a high potential energy site due to orientational hardening, which increases the values according to static stress applied.

The retardation time $\left(\tau=\eta_{K} / E_{K}\right)$ taken to produce $63.2 \%$ of total deformation in the Kelvin unit was calculated (3). It can be noted that the values decreases with temperature, when the same stress is analyzed. This is due higher molecular mobility as earlier explained. However, when compared different stresses the values where higher due more molecular cooperativity necessary.

\subsubsection{Permanent viscous flow $\eta_{M}$}

This parameter is, probably, the most important one since it represents the irrecoverable creep i.e, it is a measure of the amount of residual strain left in the specimen after repeated creep and recovery, relative to the amount of stress applied. It showed to be very sensitive to temperature effects. Since $\eta_{M}$ is associated with viscous part of the material, it can be related to interfacial properties and results from $\tan \delta$ peak height. The creep behavior is directly associated with the interface property: a better interface results in lower creep. Also, this parameter can be related to the damage of oriented non-crystalline regions 9 . Similarly to the other Burger's model parameters, this constant got lower with increasing temperature, due to the greater mobility of the molecular chains at higher temperatures. Generally, the parameter seems to increase with stress level. A greater portion of molecules are necessary for cooperativity when higher stress is applied, consequently, a higher deformation in time is obtained which increases the permanent viscous flow.

\subsection{Weibull model}

As the model suggests ${ }^{8}$, latches are activated under time and the triggering time of each latch is dependent on the 
stiffness of the correspondent spring and viscosity of the dashpot. Under creep conditions, triggering times would be reduced as creep load is increased, thereby increasing the strain rate. The failure of the elements in a system, the incremental step argument can be related to the relaxation in amorphous materials, which is represented by time-dependent phenomena from Kohlrausch-Williams-Watts (KWW) function. Originally, in spite of to be represented by dielectric decays, this function is identical in form to the Weibull one. i.e., an empirical "stretched exponential" function. So, it allows characterizing creep in amorphous polymers.

It was chosen the temperature of $30^{\circ} \mathrm{C}$ at different stress levels for represent Weibull model. Parameters from Eq. 4 can be found in Table 3. In general, it can be observed a trend from the results.

Table 3. Weibull parameters for VARTM composites.

\begin{tabular}{cccc}
\hline \multirow{2}{*}{ Parameters } & \multicolumn{3}{c}{ Stress (MPa) } \\
\cline { 2 - 4 } & 2 & 5 & 10 \\
\hline$\varepsilon_{i}(\%) \times 10^{-3}$ & $\begin{array}{c}1.36 \pm 7.76 \\
\times 10^{-2}\end{array}$ & $2.17 \pm 0.145$ & $4.25 \pm 0.138$ \\
$\varepsilon_{c}(\%) \times 10^{-4}$ & $8.84 \pm 1.01$ & $21.60 \pm 5.3$ & $47.50 \pm 8.34$ \\
$(\mathrm{sec})$ & $67.63 \pm 11.97$ & $504.43 \pm 339.97$ & $724.41 \pm 291.27$ \\
$\beta_{c}$ & $0.426 \pm 0.066$ & $0.437 \pm 0.108$ & $0.695 \pm 0.114$ \\
$\mathrm{R}^{2}$ & 0.9984 & 0.9969 & 0.9968 \\
\hline
\end{tabular}

$\varepsilon_{i}$ is referred to the instantaneous strain from the initial application of the load. It can be observed that the values increase by stress level applied, which is expected since the instantaneous strain from initial application of the load for causing creep is higher. The number of latches activated is higher, i.e., the number of chain segments which are activated together increase with static stress level. $\varepsilon_{c}$ is referred to the cumulative number of activations with time, and it can be observed, again, higher values with stress level. Since the KWW function is a good approximation to the potential energy barrier as proposed by Eyring ${ }^{23}$, the results of the present study suggest that the applied load will cause the progressive activation of latch elements, higher stress levels will promote a higher number of these latches activated over time. In molecular terms, it can be associated with a higher number of chain segments which "work together" cooperatively to overcome any energetic barrier for molecular motion, i.e., the viscoelastic changes are suggested to occur though incremental jumps in which segments of molecules jumps between positions of relative stability. $\eta_{c}$ is referred to the characteristic time life, where $63.2 \%$ of the elements have failed. The higher values obtained with static stress level applied is due higher $\varepsilon_{c}$ values, when a higher portion of the chain segments is necessary for activation due higher stress level imposed, which it takes longer times. $\beta_{c}$ is the shape parameter format. It can be observed that the values increasing with static stress level. In mechanical terms (latch model), it means that slower strain rate allows a greater proportion of latches with longer trigger times to become activated during creep cycle. Since the formula can be approximated to a dependence level derived from a model describing in principle the kinetics of any process ${ }^{24}$, it can be used for describing the kinetics of relaxation of any structural order parameter in terms of time dependent relaxation times. In molecular terms, it is associated with higher relaxation times promoted by a higher number of segments which need to be activated together. Consequently, the incremental step for failure of the chain segments occurs for a more homogeneous manner, since the stretched exponential function is nearer to unity for higher stress levels.

\section{Conclusions}

In this study, viscoelastic properties of an epoxy carbon/ fiber composite obtained by VARTM were studied. By DMA, it was possible to verify the relationship of temperaturedependency of the composite. Creep measurements were based on dynamic mechanical runs. As expected, higher temperatures lead out to a higher instantaneous deformation due to a higher molecular mobility achievement. By Burger's parameters, it were also verified the influence of stress and temperature on the viscous flow, related to molecules cooperativity. In general, temperature and stress increased viscous flow, leading to higher molecular chains relaxation and resulting in poorer properties. Findley's parameters showed a great dependency of temperature, since the creep strain increases. The study of stress-dependency by Weibull shape parameter $\beta$ showed the cooperativity caused by different chain segments movement, corroborating with previous conclusions.

\section{Acknowledgements}

The authors would like to thanks to CAPES, UCS, UNESP, $\mathrm{CNPq}$ for contributions and financial support.

\section{References}

1. Brocks T, Shiino MY, Cioffi MOH, Voorwald HJC, Caporalli Filho A. Experimental RTM manufacturing analysis of carbon/ epoxy composites for aerospace application: non-crimp and woven fabric differences. Materials Research. 2013;16(5):11751182

2. Botelho EC, Silva RA, Pardini LC, Rezende MC. A review on the development and properties of continuous fiber/epoxy/ aluminum hybrid composites for aircraft structures. Materials Research. 2006;9(3):247-256. 
3. Arteiro A, Catalanotti G, Xavier J, Camanho PP. Notched response of non-crimp fabric thin-ply laminates. Composite Science and Technology. 2013;79:97-114.

4. Vallons K, Lomov SV, Verpoest I. Fatigue and post-fatigue behaviour of carbon/epoxy non-crimp fabric composites. Composites Part A: Applied Science and Manufacturing. 2009;40(3):251-259.

5. Vallons K, Behaeghe A, Lomov SV, Verpoest I. Impact and post-impact properties of a carbon fibre non-crimp fabric and a twill weave composite. Composites Part A: Applied Science and Manufacturing. 2010;41(8):1019-1026.

6. Williams C, Summerscales J, Grove S. Resin Infusion under Flexible Tooling (RIFT): a review. Composites Part A: Applied Science and Manufacturing. 1996;27(7):517-524.

7. Ornaghi HL Jr, da Silva HSP, Zattera AJ, Amico SC. Dynamic mechanical properties of curaua composites. Journal of Applied Polymer Science. 2012;125(S2):E110-E116.

8. Fancey KS. A mechanical model for creep, recovery and stress relaxation in polymeric materials. Journal of Materials Science. 2005;40(18):4827-4831.

9. Yang JL, Zhang Z, Schlarb AK, Friederich K. On the characterization of tensile creep resistance of polyamide 66 nanocomposites. Part II: Modeling and prediction of long-term performance. Polymer. 2006;47(19):6745-6758.

10. Militký J, Jabbar A. Comparative evaluation of fiber treatments on the creep behavior of jute/green epoxy composites. Composites Part B: Engineering. 2015;80:361-368.

11. Almeida JHS Jr., Ornaghi HL Jr., Lorandi NP, Bregolin BP, Amico SC. Creep and interfacial behavior of carbon fiber reinforced epoxy filament wound laminates. Polymer Composites. 2017. In Press. DOI: 10.1002/pc.24537

12. Goertzen WK, Kessler MR. Creep behavior of carbon fiber/ epoxy matrix composites. Materials Science and Engineering: A. 2006;421(1-2):217-225.

13. Fliegener S, Hohe J, Gumbsch P. The creep behavior of long fiber reinforced thermoplastics examined by microstructural simulations. Composite Science and Technology. 2016;131:1-11.
14. Wu CM, Lin PC, Murakami R. Long-term creep behavior of self-reinforced PET composites. Express Polymer Letters. 2017;11(10):820-831.

15. Findley WN, Lai JS, Onaran K. NonLinear Creep at Constant Stress and Relaxation at Constant Strain. In: Findley WN, Lai JS, Onaran K. Creep and Relaxation of Nonlinear Viscoelastic Materials With an Introduction to Linear Viscoelasticity. Amsterdam: North-Holland; 1976, p. 176-208.

16. Ornaghi HL Jr., Bolner AS, Fiorio R, AJ Zattera AJ, Amico SC. Mechanical and dynamic mechanical analysis of hybrid composites molded by resin transfer molding. Journal of Applied Polymer Science. 2010;118(2):887-896.

17. Mallarino S, Chailan JF, Vernet JL. Glass fibre sizing effect on dynamic mechanical properties of cyanate ester composites I. Single frequency investigations. European Polymer Journal. 2005;41(8):1804-1811.

18. Deng S, Hou M, Ye L. Temperature-dependent elastic moduli of epoxies measured by DMA and their correlations to mechanical testing data. Polymer Testing. 2007;26(6):803-813.

19. Ferry JD. Viscoelastic Properties of Polymers. $3^{\text {rd }}$ ed. New York: John Wiley \& Sons; 1980. p 46-47.

20. Lorandi NP, Cioffi MOH, Ornaghi HL Jr. Dynamic Mechanical Analysis (DMA) of Polymeric Composite Materials. Scientia cum Industria. 2016;4(13):48-60.

21. Ornaghi HL Jr., Zattera AJ, Amico SC. Dynamic mechanical properties and correlation with dynamic fragility of sisal reinforced composites. Polymer Composites. 2015;36(1):161-166.

22. Georgiopoulos P, Kontou E, Christopoulos A. Short-term creep behavior of a biodegradable polymer reinforced with woodfibers. Composites Part B: Engineering. 2015;80:134-144.

23. Glasstone S, Laidlev KJ, Eyring $\mathrm{H}$. The theory of rate processes: the kinetics of chemical reactions, viscosity, diffusion and electrochemical phenomena. $1^{\text {st }}$ ed. New York: McGraw-Hill; 1941.

24. Dobreva A, Gutzow I, Schmelzer J. Stress and time dependence of relaxation and the Kohlrausch stretched exponent formula. Journal of Non-Crystalline Solids. 1997;209(3):257-263. 